\title{
Dynamic and reversibility of heterochromatic gene silencing in human dis- ease
}

\author{
Giuseppe ZARDO ${ }^{1,2}$, Francesco FAZI ${ }^{2,3}$, Lorena TRAVAGLINI ${ }^{2,3}$, Clara NERVI ${ }^{2,3, *}$ \\ 'Departments of Cellular Biotechnology and Hematology, University of Rome "La Sapienza", Via di Castel Romano 100, \\ Rome 00128, Italy \\ ${ }^{2}$ San Raffaele Bio-medical Science Park of Rome, Via di Castel Romano 100, Rome 00128, Italy \\ ${ }^{3}$ Departments of Histology and Medical Embryology, University of Rome "La Sapienza", Via di Castel Romano 100, Rome \\ 00128 , Italy
}

\begin{abstract}
In eukaryotic organisms cellular fate and tissue specific gene expression are regulated by the activity of proteins known as transcription factors that by interacting with specific DNA sequences direct the activation or repression of target genes. The post genomic era has shown that transcription factors are not the unique key regulators of gene expression. Epigenetic mechanisms such as DNA methylation, post-translational modifications of histone proteins, remodeling of nucleosomes and expression of small regulatory RNAs also contribute to regulation of gene expression, determination of cell and tissue specificity and assurance of inheritance of gene expression levels. The relevant contribution of epigenetic mechanisms to a proper cellular function is highlighted by the effects of their deregulation that cooperate with genetic alterations to the development of various diseases and to the establishment and progression of tumors.
\end{abstract}

Keywords: DNA methylation, chromatin remodeling, epigenetics, retinoic acid, acute myeloid leukemia.

\section{MOLECULAR MECHANISM OF DNA METH- YLATION REACTION}

Among all epigenetic mechanisms involved in gene expression regulation, DNA methylation has been the most widely studied subject. DNA methylation results from the transfer of a methyl group from a methyl donor substrate, namely S-adenosyl-L-methionine (AdoMet), to the 5' position of a cytosine in the palindrome dinucleotide $\mathrm{CpG}$ [1]. Non CpG DNA methylation has also been observed in eukaryotic genomes on $\mathrm{CpNpG}$ or $\mathrm{CpA}$ and $\mathrm{CpT}$ sequences [2], which account for almost $20 \%$ of the global methylation in mouse embryonic stem cells [3]. The function of non $\mathrm{CpG}$ methylation however needs to be further elucidated. To date, cytosine methylation represents a unique chromatin modification that can be faithfully propagated through cell divisions [4]. In eukaryotes the $\mathrm{CpG}$ methylation reaction is catalyzed by enzymes known

\footnotetext{
*Correspondence: Clara NERVI

Tel: +39-06-80319049/52; Fax: +39-06-80319054;

E-mail:clara.nervi@uniroma1.it
}

as DNA cytosine-5-methyltransferases (DNMTs) $[5,6]$ (Fig. 1) including:

\section{DNA cytosine-5-methyltransferase 1}

DNA cytosine-5-methyltransferase 1 (DNMT1), known as a maintenance methylase, copies the parental DNA methylation patterns onto daughter DNA strand during replication. The maintenance role of pre-existing DNA methylation patterns is attributed to DNMT1 based on its activity on hemimethylated substrate and its nuclear localization during DNA replication. DNMT1 activity is 5-50 folds higher in presence of a hemimethylated substrate as compared to that in the presence of an unmethylated DNA sequence $[7,8]$. Nonetheless, the specific activity of DNMT1 on unmethylated DNA substrates is greater than that of DNMT3A and DNMT3B, which are considered as "de novo" DNMTs instead [6].

Moreover, DNMT1 localizes in association with PCNA proteins to the replication foci during the $S$ phase [9]. DNMT1 through its amino terminal domain interacts with the DNMT1 associated protein (DMAP1), histone deacetylases (HDACs) and retinoblastoma protein $(\mathrm{Rb})[10$ - 


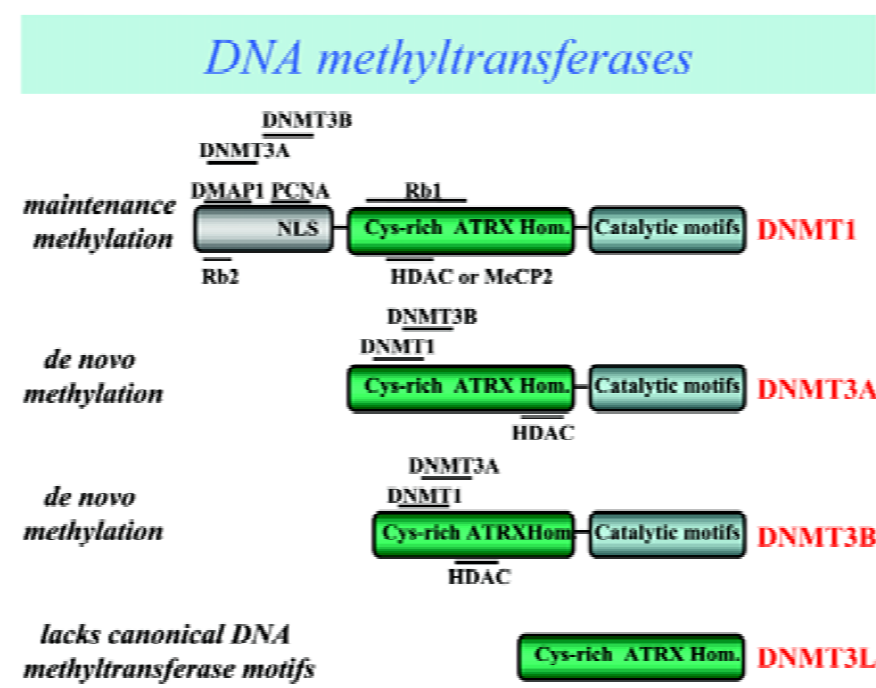

Fig. 1 Schematic representation of DNA-methyltransferases functional/structural domains in relation to their activities. (-) indicates the binding sites for: DMAP1, PCNA, Rb, HDAC and MeCP2. The nuclear localization signal (NLS), the conserved cysteine-rich region (Cys-rich), which shares homology with the X-linked ATRX gene of the SNF2/SWI family (ATRX Hom) and the catalytic motifs are also indicated.

12]. DNMT1 is constitutively expressed in proliferating cells and ubiquitously in somatic tissues throughout mammalian development. A number of studies have been conducted to evaluate the biological consequences of losing DNMT1 activity. In $1992 \mathrm{Li}$ et al [13] have shown that insertion of a mutant DNMT1 gene into mouse germ line produces a recessive lethal phenotype. The $\mathrm{m} 5 \mathrm{C}$ levels in homozygous mutant mouse ES cells were one-third of that in wild-type cells but the cells were still viable. In addition, loss of a functional DNMT1 also determines the following:

1) mice or embryonic stem (ES) cells homozygous for null alleles of DNMT1 are generally demethylated, although there is a basal methylation level [14];

2) biallelic expression of most imprinted genes in homozygous embryos [15];

3 ) an increase of mutation rates for exogenous marker genes in ES cells and an increase of losing heterozygosity due to mitotic recombination [16];

4) enhanced microsatellite instability in mouse embryonic stem cells [17];

5) demethylation and expression of transposons of the intracisternal A particle (IAP) class, the LTR retroposons [18-20] attributing to the specific role of DNMT1 in gene silencing on transposable elements;

6) very high rates of lethal T-cell lymphomas [21].

\section{DNA cytosine-5-methyltransferase $3 A$ and $3 B$}

DNA cytosine-5-methyltransferase $3 \mathrm{~A}$ and $3 \mathrm{~B}$ (DNMT3A and 3B), known as de novo methylases, methylate unmethylated $\mathrm{CpG}$ dinucleotides [6]. DNMT3A and DNMT3B are highly expressed in ES cells, early embryos, and developing germ cells where de novo methylation takes place, but expressed at lower rate in somatic tissues of postnatal animals [22]. These enzymes have no preference for hemimethylated DNA [22, 23] and can methylate de novo unmethylated $\mathrm{CpGs}$ when ectopically expressed in mammalian cells or transgenic flies [24, 25]. DNMT3A has also been reported to methylate $\mathrm{CpA}$ sites [3]. DNMT3A-deficient mice survive to adult but die early in adulthood with global methylation patterns maintained [6]. DNMT3A-/- DNMT3B-/- double knock-out mouse embryos die at $8.5 \mathrm{dpc}$ with global demethylation in genomes [6]. Point mutations in human DNMT3B are responsible for the autosomal recessive human disorder known as ICF (immunodeficiency, centromere instability, and facial anomalies) syndrome [23].

\section{DNA cytosine-5-methyltrasferase 3 Like}

DNA cytosine-5-methyltrasferase3 Like (DNMT3L) was originally identified by database analysis of the human genome sequences and subsequently a mouse homolog was characterized [26-28]. This enzyme lacks amino acids essential for the catalytic activity of methyltransferase. DNMT3L is thus unable to perform enzymatic activity. The gene is however essential for the establishment of maternal methylation imprints and for the correct expression of maternally imprinted genes [28]. A mechanism of forming protein complex with DNMT3A and $3 \mathrm{~B}$ and thus modulating the enzymatic activities of DNMT3A and $3 \mathrm{~B}$ is probably involved [27, 29].

The DNMT1, DNMT3A and DNMT3B cooperate to establish and maintain DNA methylation patterns. Genetic and biochemical analyses have shown that double knockout of DNMT1 and DNMT3b diminishes most of the genomic methylation while single knock-out has limited effect on DNA methylation pattern [30]; that DNMT1 alone is insufficient to restore DNA methylation after 5-azaCdR treatment [31] and that DNMT1 can form complex with DNMT3A and 3B [32] (Fig. 1).

\section{CPG DINUCLEOTIDES LOCATION AND FUNC- TION}

DNA methylation levels are not equal in eukaryotic genomes with a great disparity found in the four eukaryotic groups (protists, plants, fungi, and animals). Furthermore, CpG dinucleotides are not equally distributed along the genome; most of them are gathered in regions named " $\mathrm{CpG}$ islands" [33]. 
In mammals $\mathrm{CpG}$ islands ranging from 0.4 to $4 \mathrm{~Kb}$ in length, have CG content higher than 55\%, which is five folds higher than that in bulk DNA and are unmethylated [34]. The unmethylated status of $\mathrm{CpG}$ islands is preserved during replication and maintained during following cell cycles despite of their accessible localization in decondensed chromatin for enzymes and transcription factor. Although maintenance of unmethylated $\mathrm{CpG}$ status has been studied by several groups [35-40], the detailed mechanism is still not very clear. $\mathrm{CpG}$ islands usually span the 5 '-end region-promoter, untranslated region and exon 1 of $40 \%$ mammalian genes [41].

The $\mathrm{CpG}$ dinucleotides dispersed along the genome and located in non regulatory DNA regions on the other hand are largely methylated. A great majority of these CpGs resides in transposable elements, which are interspersed repeated sequences that constitute more than $45 \%$ of the human genome [42]. Transposable elements can be reactivated by demethylation of methylated CpGs [20, 43]. These observations strongly suggest a role for DNA methylation in the protection of eukaryotic genome from viral and parasitic sequences. The de-silencing of transposable elements affects the genome stability and the expression of genes through different mechanisms including the activation of RNAi pathways [8, 44].

DNA methylation patterns are established during embryonic development and should be maintained unchanged during adult life for proper cellular function [45]. Germ cells of females are less methylated than those of males, and after fertilization gamete methylation patterns are erased by a wave of genome wide demethylation [46] that is active in the sperm derived male pronucleus and passive in the female pronucleus [47]. During the implantation stage, methylation patterns are established by a wave of de novo methylation and they probably serve to compartmentalize the genome into transcriptionally active and inactive zones. The methylation status of $\mathrm{CpG}$ sites within gene regulatory regions strongly affects the expression of the downstream genes. In particular the unmethylated status of a 5' promoter $\mathrm{CpG}$ island is associated with the transcription of the downstream gene, whereas $\mathrm{CpG}$ island methylation inhibits gene transcription; genes on inactive $\mathrm{X}$ chromosome, imprinted genes and tumor suppressor genes in tumors are silenced and have regulatory elements largely methylated.

If methylation of regulatory $\mathrm{CpG}$ islands is critical for gene expression, intragenic methylation in mammalian genes never blocks transcription and RNA elongation. Very recently Lorincz et al [4] have shown that the efficiency of transcription can be seriously affected by methylation of non regulatory CpGs dispersed in the body of the gene.

We may assert that DNA methylation exerts its func- tions through an inhibitory effect on gene expression. For instance, functioning DNA methylation machinery is fundamental for a correct expression of imprinted genes. While the great majority of genes are expressed from both maternal and paternal alleles, few genes are specifically expressed only from one allele. In these cases the promoter $\mathrm{CpG}$ islands associated with the silent genes are largely methylated [48]. In addition, in mammalian females the inactivation of $\mathrm{X}$-chromosome is dependent on DNA methylation, $\mathrm{CpGs}$ sites in promoters of the genes on the inactive $\mathrm{X}$ chromosome are methylated and the genes are transcriptionally silent [49]. A role for DNA methylation in tissue specific gene expression and differentiation has also been proposed. First data on DNA methylation and tissue specificity were obtained by Walsh and Bestor [18]. These Authors investigated the 5' methylation status of seven tissue-specific genes and found no correlation with tissue-specific expression. Recent results, mostly obtained by genome wide approaches to study DNA methylation, do confirm a role for DNA methylation in tissue specific gene expression. One of these genome wide approaches known as Restriction Landmark Genome Scanning (RLGS) has enabled to identify in six mice tissues a large number of differentially methylated regions (TDMs) which exhibit a tissue-specific expression pattern that is consistent with methylation status and a role in tissue differentiation [50]. Furthermore, Ching et al [51], by using BAC microarray have identified $\mathrm{CpG}$ island loci that are methylated in a tissue-specific manner in normal human tissues, including SHANK3 whose differential methylation in different tissues was also evolutionary conserved.

\section{MECHANISM OF GENE SILENCING BY DNA METHYLATION}

Two mechanisms have been proposed to explain the inhibitory effect of $\mathrm{CpG}$ methylation on gene expression. First, CpGs methylation inhibits binding of transcription factors to their $\mathrm{CpG}$ containing recognition sites [52]. The second mechanism proposes the involvement of proteins with high affinity for methylated CpGs. MeCP1 and MeCP2 (methyl $\mathrm{CpG}$ binding protein 1 and 2) were the first proteins with high affinity for methylated DNA containing a "methyl binding domain" (MBD) described [53]. MeCP1 targets DNA sequences containing a minimum number of methylated $\mathrm{CpGs}$, whereas MeCP2 binds a single symmetrically methylated $\mathrm{CpG}$ pair. Both proteins were found to bind chromosomes at sites known to contain methylated DNA. Mutations in MeCP2 cause the human female neurodevelopmental disorder called Rett syndrome and a closely related disorder in $\mathrm{MeCP} 2$ mutant mice [54]. On the basis of the ability to bind methylated sequences in vitro other methyl $\mathrm{CpG}$ binding proteins have been identified: 

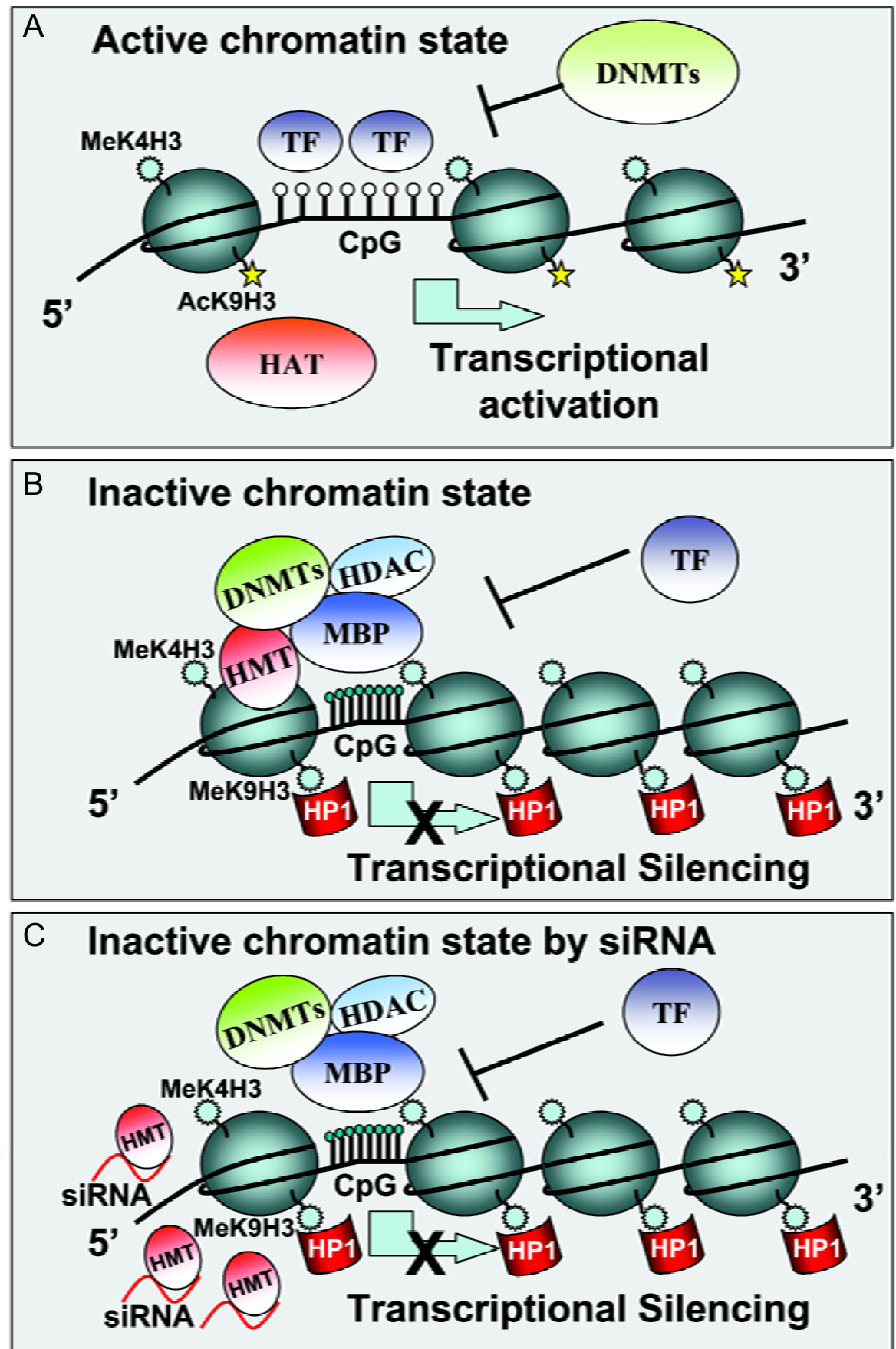

Fig. 2 Epigenetic modifications leading to gene transcriptonal regulation. TF: transcriptional factors; DNMT: DNA methyltransferase; HAT: Histone acetyltransferase; HDAC: Histone deacetylase; MBP: Methyl-CpG binding protein; HMT: Histone methyltransferase. Modifications of the histone H3 tails, Lys4 (K4) and Lys9 (K9), are indicated. Ac: acetylation; Me: methylation.

MBD1, MBD2, MBD3, MBD4 and Kaiso. MBD1 was first identified as a methyl binding protein and it has been recently shown as able to bind $\mathrm{CpG}$ sequences independently of the methylation status [55]. MBD2 is part of the MeCP1 protein complex [56]. MBD3 binds methylated probes in vitro but this interaction is not competed by an excess of methylated cold competitor making its ability to interact specifically with methylated sequences questionable [57]. MDB4 turns out to be a DNA T:G mismatch repair enzyme and may function to minimize mutation at 
methyl-CpG sites [58]. The transcriptional repressor Kaiso, which binds methyl-CpGs through a zinc-finger motif [59], recognizes methylated DNA regions containing at least two methylated CpGs. The knock-down of Kaiso leads to premature zygotic gene activation and subsequent developmental arrest and apoptosis, mimicking the phenotype of hypomethylated embryos deficient in DNMT1 [60]. In HeLa cells, Kaiso is coupled with the NCoR corepressor to mediate DNA methylation-dependent gene silencing [61].

\section{THE CONNECTION BETWEEN DNA MET HY- LATION AND OTHER EPIGENETIC MECHA- NISMS OF GENE SILENCING}

Despite the exact cascade of events leading to remodeling of chromatin structure and gene inactivation or activation, DNA methylation, histone acetylation, deacetylation and histone methylation all work together to build up chromatin structures which coordinately may shift from a transcriptional permissive state to a transcriptional inactive state and vice versa (Fig. 2). The methyl $\mathrm{CpG}$ binding proteins exert their function as transcriptional repressors via chromatin remodeling. These proteins are often part of larger repressor complexes as NuRD, NoRC, $\mathrm{mSin} 3 \mathrm{~A}$ and SWI-SNF, that recruit HDACs [56, 62-67] and histone methyltransefrases (HMTs) [68] on methylated targeted sequences. Acetylation of the $\alpha$ amino groups of evolutionary conserved lysine residues present on nucleosomal histone $\mathrm{H} 3$ and $\mathrm{H} 4$ is catalyzed by the histone acetyl transferases (HAT). The level of histone acetylation at these sites depends on the equilibrium between the histone acetyl transferase activity and histone deacetylase activity [69]. Methylation of nucleosomal histones occurs on lysine and arginine residues and the reaction is catalyzed by the HMTs [70]. Both the HDAC and HMT activities determine changes in the post translational modification of histone tails thus promoting the shift from a transcriptionally permissive chromatin state to a transcriptionally inactive chromatin state (reviewed in [71]) as schematically represented in Fig. 2.

In mammalians, the decondensed transcriptionally active euchromatin is enriched for lysine 9 (K9) acetylated histone $\mathrm{H} 3$ and lysine 4 (K4) methylated histone H3 (mainly in lower eukaryotes), whereas K9 deacetylation, K9 (di and tri-methylation) and K27 methylation of H3 histone are common marks of a condensed transcriptionally inactive heterochromatin [70]. Furthermore, methylated K9 histone $\mathrm{H} 3$ recruits the heterochromatin protein 1 (HP1) allowing the stabilization of an inactive condensed chromatin [70]. A very intriguing role for $\mathrm{K} 9 \mathrm{H} 3$ methylation has been suggested by experiments conducted on Neurospora crassa, Arabidopsis thaliana and mammalians. In Neurospora crassa, mutations in Su(var)3-9 H3K9 methyl- transferases (dim-5) gene are responsible for a genome wide demethylation of methylated CpGs [72]. The same result was obtained in A. thaliana by mutations of Kryptonite gene, a relative of $\mathrm{Su}(\mathrm{var}) \mathrm{H} 3-\mathrm{K} 9$ methyltransferase, in this case the $\mathrm{CpNpG}$ methylated sequences were largely demethylated while methylation status of CpGs remained unchanged [73]. In mice, mutation of the two $\mathrm{Su}(\mathrm{var}) 3-9$ homologs has a relatively minor effect on cytosine methylation and causes partial demethylation of major satellite regions of pericentromeric heterochromatin [74]. These data suggest a clear dependence of DNA methylation on histone $\mathrm{H} 3$ methylation.

Literature provides evidences that support the current debate on the exact cascade of events leading to gene inactivation through chromatin remodeling. The DNA methylation may represent the in cis input able to induce the recruitment of chromatin remodeling factors and the following shift of chromatin structure from a transcriptional active to a transcriptional inactive state. On the other hand $\mathrm{H} 3 \mathrm{~K} 9$ methylation may represent the in trans input as shown by his role in targeting DNA methylation in fungi, planta and mammalians. In this case the histone modifications and the successive chromatin remodeling precede changes in DNA methylation pattern.

\section{PUTATIVE REGULATORY ROLE OF THE RNAI PATHWAYS IN THE EPIGENETIC CONTROL OF GENE EXPRESSION.}

The idea that RNAi pathways can target epigenetic modifications, such as DNA methylation and histone methylation, to genomic specific regions has gained a tremendous attention in the last decade. The first data showing a connection between DNA methylation and RNAi pathway were obtained in viroid-infected tobacco plants [75]. In viroid infected plants the cDNA copies integrated into genome undergo DNA methylation directed by RNA. Subsequenly, it was shown that this de novo methylation activity required formation of dsRNA and of processed siRNA [76]. siRNAs direct DNA methyltransferases (MET1, CMT3), histone methyltransferases (DDM1, Kryptonite) and histone deacetylases onto targets promoter regions and induce transcriptional gene silencing in plants $[77,78]$. Although not all the components of the RNAdependent DNA methylation machinery have been identified, it seems that a similar process exists in mammalian cells.

The expression of elongation factor 1 alpha (EF1A) promoter-green fluorescent protein (GFP) integrated into the genome of human 293FT cells is inhibited following the transfection of EF1A homologous sequence EF52 siRNA and is associated with DNA methylation status shown by methylation sensitive restriction enzyme. This inhibition is 
sensitive to 5-azacitidine (DNMTs inhibitor) and tricostatin A (deacetylase inhibitor) treatments. The transcription of endogenous EF1A is also regulated by and associated with DNA methylation of promoter region [79].

siRNAs targeted to promoter $\mathrm{CpG}$ island of E-cadherin gene in MCF-7 and normal mammary epithelial cells induce DNA methylation, histone $\mathrm{H} 3 \mathrm{~K} 9$ methylation and transcriptional repression. This siRNA mediated DNA methylation is DNMT1- or DNMT3B-dependent [80]. In addition, another group of smaller RNAs namely the microRNAs (miRNAs) might also be involved in epigenetic control of gene expression through DNA methylation and chromatin remodeling [81]. miRNAs are derived from precursors and are exported into the cytoplasm from the nucleus. Once in the cytoplasm, miRNAs interact with a target mRNA, via the RNA-induced silencing complex (RISC), to degrade the mRNA or to prevent protein translation. Bao et al [81] report an extensive study on DNA methylation in a region located more than $1 \mathrm{~Kb}$ downstream of a "miRNA-binding sites" in the PHB and PHV genes in $A$. thaliana. When the miRNAs binding sites on PHB and PHV genes are mutated, an extensive demethylation is observed within this region. The authors suggest that the demethylation facilitates gene reactivation and the methylation is mediated by miRNA [82].

\section{ABERRANT EPIGENETIC CHANGES OF DNA AND CHROMATIN IN HUMAN DISEASE}

The deregulation of epigenetic control on gene expression is frequently related to human diseases. The Rett syndrome, an X linked neurodevelopmental disorder, is linked to mutations in the $\mathrm{MeCP} 2$ gene. Patients affected by ICF syndrome carry mutations in the methyltransferase gene DNMT3B. The Beckwith-Wiedemann syndrome, the
Prader-Willi syndrome and the Angelman syndrome are human diseases caused by deregulation of imprinted genes or by epigenetic defects (reviewed in [83]).

Epigenetics alterations may also have effects on the onset of mental diseases such as schizophrenia and affective disorders [83]. Moreover, studies accumulated during the past two decades have proven that epigenetics is indeed involved in controlling and regulating cancer formation and progression. Aberrant DNA methylation patterns are extremely common in all types of tumors. In considerations of: i) the role of DNA methylation in the regulation of gene expression and in the maintenance of genome stability; ii) the aberrant methylation patterns observed in human specimens ahead of the onset of malignancy [84, 85]; iii) the sensitivity of cancer patients to DNMTs and HDACs inhibitors, it is plausible that an epigenetic deregulation in general and altered DNA methylation pattern in particular are not a consequence but rather a primary cause of malignant state [86].

DNA methylation machinery has been damaged seriously in cancer cells when largely aberrantly hypomethylated genomic sequences coexist with aberrantly hypermethylated sequences creating one of the most intriguing biological paradoxes [87]. The causes of such dichotomous are however not known.

Cancer cells undergo significant DNA hypomethylation [88] that involves methylated CpGs in non regulatory regions such as intragenic sequences and repetitive elements. Mice carrying a hypomorphic DNMT1 allele have reduced DNMT1 expression up to $10 \%$ of the wild type levels and have a substantial genome-wide hypomethylation in all tissues. These mice develop aggressive $\mathrm{T}$ cell lymphomas that display a high frequency of chromosome 15 trisomy $[21,89]$. Hypomethylated cells carrying a hypomorphic

Tab. 1 Functional classification of putative/established tumor suppressor genes found hypermethylated and silenced in cancer cells

\begin{tabular}{|c|c|}
\hline Function & Gene \\
\hline Cell cycle regulation & $p 16^{\text {ink } 4 a}, p 15^{\text {ink } 4 b}, R b, p 27, p 73,14-3-3 \sigma$ \\
\hline Apoptosis & DAPkinase, Caspase 8, TMS1 \\
\hline DNA repair & $h M L H 1, M G M T, B R C A 1$ \\
\hline Hormone response & $E R, P R, A R, P R L R, R A R \mathrm{~b} 2$ \\
\hline Metastasis/invasion & CDH1, CDH13, TIMP3, Maspin, FAT \\
\hline Signal transduction & $A P C, P T E N, R A S S F 1 A, N O R E 1 A, S T K 11, L K B 1$ \\
\hline Drug resistance/detoxification & $G S T P 1, M D R 1$ \\
\hline Transcription/transcription factors & VHL, HIC-1, GATA4, GATA5 \\
\hline Angiogenesis & $T H B S-1$ \\
\hline Inflammation & $C O X-2$ \\
\hline Differentiation & MYOD1, PAX6, WT1 \\
\hline Involved in other biological processes & $C A C N A 1 G, C A L C A, F H I T, T E R T, T P E F, C S P G 2$ \\
\hline
\end{tabular}


allele of DNMT1 undergo an increase in loss of heterozygosity $(\mathrm{LOH})$ rate, in consistent with the hypothesis that hypomethylation promotes tumor development in NPcis mice by increasing the rate of $\mathrm{LOH}$ [89]. In addition, patients having DNMT3B gene mutated in the germ line are prone to chromosome instability [21]. These results indicate that the tumorigenic effect of DNA hypomethylation might reside in an increase of chromosomal instability induced by the loss of methylation in specific chromosomal areas. Furthermore, loss of imprinting of specific DNA sequences may be attributed to DNA hypomethylation. Loss of imprinting of H19/IGF-2 locus in some childhood tumors is a typical example (reviewed in [90]).

DNA hypomethylation is also responsible for reactivation of transposable elements and silenced viral sequences, via erratic insertion of DNA fragments into transcribed regions or through a siRNA pathway [9, 92]. Moreover, a diet deficient in folate, a vitamin involved in AdoMet metabolism, is associated with DNA hypomethylation and hepatocarcinogenesis in murine (reviewed in [93]).

It has been hypothesized that DNA hypomethylation might also lead to re-activation of silenced oncogenes, through promoter demethylation. However, there is no supporting data available yet. Nevertheless, we cannot exclude the possibility that the promoter demethylation and the de-silencing of certain tissue specific genes at wrong place and wrong time might induce an oncogenic effect.

It is still a debate for the role of aberrant DNA hypomethylation in cancer formation. On the other hand, the role of aberrant DNA hypermethylation in tumor formation and progression has already been well established. Aberrant DNA hypermethylation can contribute tumorigenesis by different mechanisms. One way is to induce mutational hotspots. Methylation of cytosine in $\mathrm{CpG}$ dinucleotide favorites spontaneous deamination of cytosine that leads to $\mathrm{C}$ to $\mathrm{T}$ point mutations in transcripts as well as in promoter regions [94]. Furthermore, a less efficient mismatch repair of $\mathrm{T} / \mathrm{G}$ can increase the rate of $\mathrm{C}$ to $\mathrm{T}$ point mutations [95].

Aberrant DNA methylation of regulatory $\mathrm{CpG}$ islands is frequently associated with gene silencing. Loss of expression of important tumor suppressor genes in cancer cells is generally attributed to genetic lesions such as mutations and deletions as well as aberrant methylation in promoter or regulatory regions (Tab. 1).

Homozygous methylation of promoter $\mathrm{CpG}$ islands is the prevailing mechanism of gene silencing in the majority of genes inactivated by epigenetic mechanisms. However, as shown by Zardo et al [96], epigenetic and genetic mechanisms may converge on specific sites and contribute to gene silencing. The aberrant DNA hypermethylation in cancer cell shows fascinating and peculiar features that might be exploited for diagnostic, prognostic, and therapeutic purposes. For example, not all the genes are prone to DNA hypermethylation in a similar way. Some genes seem to undergo aberrant DNA hypermethylation more easily and frequently than others in the same type of tumor. Other genes undergo aberrant DNA hypermethylation in a tumor type-dependent manner. Costello et al [97] by using genome wide approaches have demonstrated that aberrant DNA methylation patterns are tumor type specific rather than random. The specific patterns of aberrant DNA hypermethylation (CIMP) could be used as indicators to identify and characterize different histological subtypes of the same tumor and to predict the chemo-sensitivity of cancer and the duration of patient survival.

\section{TARGETING HETEROCHROMATIC GENE SILENCING AS NOVEL THERAPEUTICAL APPROACH FOR NEOPLASIA}

Functional deregulation of HATs, HDACs, HMTs, DNMTs or MBDs results in abnormal transcriptional regulation of target genes that are relevant to the transformation process $[98,99]$. Heritable changes in gene expression due to epigenetic modifications of chromatin by HATs, HDACs, DNMTs offer a mechanism by which upstream signaling pathways can converge to common targets associated with neoplasia. In contrast to genetic cancer causes, all these aberrant epigentic changes can be reversed by drugs targeting chromatin regulators in cells. This highlights chromatin regulators as novel targets for the development of anti-neoplastic drugs, which might induce lasting remissions and spare the short and long term toxicity associated with high dose chemotherapy. A very well known example of the efficacy of transcriptional/differentiation therapy using chromatin remodeling agents is the acute promyelocytic leukemia (APL). APL is an acute myeloid leukemia (AML) subtype, genetically characterized by chromosomal translocations involving the retinoic acid (RA) receptor $\alpha(\mathrm{RAR} \alpha)$ and generating RAR $\alpha$ fusion genes and proteins (PML/RAR $\alpha$, PLZF/RAR $\alpha, \mathrm{NPM} /$ RAR $\alpha$, NuMA/ RAR $\alpha$, Statb5/ RAR $\alpha$ etc). RAR $\alpha$ is one of the receptors that, by regulating the expression of specific genes, mediate the effect of RA (a natural derivative of Vitamin A) on development as well as on proliferation and differentiation in a number of cell systems including hematopoietic precursors [100, 101]. Biological effects of RA are mediated by two classes of RA nuclear receptors, $\mathrm{RAR} \alpha, \beta$ and $\gamma$ and retinoid $\mathrm{X}$ receptors (RXR $\alpha, \beta$ and $\gamma$ ) $[100,102]$. These receptors are members of the nuclear receptor superfamily, which includes receptors for: i) steroid hormones (e.g. estrogen and glucocorticoids); ii) non steroideal ligands (e.g. thyroid hormones), iii) diverse products of lipid metabolism, (e.g. fatty acids and prostaglan- 
dins) and a large number of so-called orphan receptors for which regulatory ligands have not been identified [100, 102]. Nuclear receptors act as ligand-inducible transcription factors that exert their effects by binding to specific response elements present in the promoters of their target genes. Heterodimerization with RXRs and interaction with a number of proteins that appear to serve co-activator or co-repressor functions, are essential for effective liganddependent transactivation of specific RA target genes by RARs [103]. In the absence of ligand, the RAR-RXR heterodimer is tightly bound to nuclear corepressors NCoR and SMRT that associate with adapter proteins like Sin3 and HDACs to form a transcriptional repressor complex at specific RA response elements (RARE) on RA target genes, which also include the RAR $\alpha$ and RAR $\beta$ themselves [100]. Deacetylation of nucleosomal core histones at these sites, induces changes of the chromatin structure (local chromatin condensation), rendering the DNA inaccessible to the RNA polymerase II transcription complex resulting in transcriptional repression. At physiological ligand concentrations (1-10 nM), RA binding releases the nuclear co-repressors and HDACs from the RAR-RXR complex, whereas transcriptional adapters and coactivators with intrinsic HAT activities (such as p300/CBP, ACTR and TIF2) are recruited to the RA response element. Hyperacetylation of histones induces structural changes in the chromatin that renders DNA accessible to the transcriptional machinery and activates the transcription of RA-target genes [69, 103, 104]. Transcriptional silencing of the RA signaling pathway is the molecular event underlying the differentiation block at the promyelocytic stage and leukemogenesis in APL [105]. The APL associated RAR $\alpha$-fusion proteins exist in vivo within oligomeric complexes $[106,107]$. This causes an aberrant recruitment of both HDAC and DNMT activities on RA-target genes [39, 105,108-112]. Paradoxically, APL also represents the most striking clinical success of a RA-based differentiation therapy in human neoplasia[105, 113]. Indeed, pharmacological doses of RA can release the HDAC repressor complex and recruit the multisubunit HAT activation complex resulting in terminal differentiation of PML/RAR $\alpha$ positive APL blasts, which account for more than $90 \%$ of APLs. Treatment with HDAC and DNMTs inhibitors is able to restore RA-effect on differentiation in RA-resistant APL blasts in vitro and in vivo [39, 111, 112]. Therefore, APL has become the molecular paradigm for therapeutic approaches utilizing chromatin remodeling agents $[105,113]$. Recent findings indicate that treatment with RA reprograms APL blasts to a non-leukemic phenotype also by inducing an early and coordinated decrease of DNMT1, DNMT3A and DNMT3B expression/activity in RA-responsive APL cell lines and primary blasts from
APL patients [112]. This correlates with de-methylation of the promoter/exon- 1 regions on RA target gene RAR $\beta 2$ in RA-responsive APL blasts cultured in vitro, and in blood samples from APL patients undergoing treatment with RA and chemotherapy. Of note, that: i) DNMT3B mRNA expression, which shows the earliest and largest decrease during normal myeloid differentiation [114], was mainly affected by RA treatment in the RA-responsive APL blasts in vitro and in vivo [112]; ii) down-regulation of DNMT expression/activity, of their occupancy at RARE sites and exon- 1 on RAR $\beta 2$ gene and of the methylation status of the RAR $\beta 2$ promoter/exon-1 are not measurable in the RAresistant NB4-MR4 cells following RA treatment [112]; iii) nanomolar concentrations of a selective RAR $\alpha$ agonist also modulated DNMT expression, and its effect is fully abolished by a RAR $\alpha$ selective antagonist [112]. Together, these results suggest a direct involvement of RA binding to either a functional PML-RAR $\alpha$ or endogenous wild type RAR $\alpha$ in the down-regulation of DNMT expression in APL. A decreased expression of DNMT1, DNMT3A and DNMT3B and granulocytic differentiation is also induced by the RAR $\alpha$ agonist AM580 in HL-60 cells, a myeloid leukemia cell line morphologically and biochemically very similar to the APL blasts lacking the $t(15 ; 17)$ but expressing a functional RAR $\alpha$ [112]. Therefore, down-regulation of DNMT expression might be a more general mechanism of RA action in cells. APL accounts for approximately $10 \%$ of AMLs, and all the other AML subtypes are not sensitive to retinoids [115]. However, an aberrant recruitment of the HDAC complexes seems not to be restricted to APL but to be present also in other AMLs. The HAT-interacting domain of the hematopoietic transcription factor AML1 is lost and replaced by the NCoR/SMRT interacting region of ETO as a result of the $t(8 ; 21)$ chromosomal translocation associated with AML-M2. Interestingly, the resulting AML1-ETO fusion protein also forms oligomeric structures that lead to transcriptional repression of AML1 an RA signaling pathways and block of myeloid differentiation $[107,116]$. Furthermore, RA and nuclear hormone receptors transcriptional coregulators with putative HAT activities (such as p300, CBP, MOZ and TIF2), are present in chromosomal rearrangements associated with some AML cases [117-121]. Moreover, a structure function analysis of the MLL-CPB product of the $\mathrm{t}(11 ; 16)$ translocation demonstrated that fusion of both the bromodomain and HAT domain of CBP to the amino portion of MLL induces full transformation and the leukemic phenotype in vivo [122]. All these findings indicate that common pathways of leukemogenesis could lie along the control of specific chromatin structures in terms of transcriptional activation or repression. Moreover, by using the DNMT and HDAC inhibitors in the presence or in the absence of RA we found 
that all these agents are active in potentiating the effect of RA on RA-responsive promoter activities, endogenous RA target genes and myeloid differentiation in either RA-responsive or RA-resistant AML blasts in vitro ([112, 123, 124] and our unpublished observations). It appears, therefore, that in AMLs different genetic alterations resulting in common patterns of deregulated gene expression may lead to differentiation block and myeloid leukemogenesis. These evidences suggest a scenario where the accessibility at specific DNA-binding sites might represent the key event for generating a chromatin code coupled to specific differentiation decision in leukemic blasts. Targeting of specific chromatin remodeling activities may represent a therapeutic strategy potentially applicable also to RA-resistant APL patients and to AML in which an aberrant transcriptional repression underlies gene silencing, maturation arrest and leukemogenesis.

\section{REFERENCES}

1 Doerfler W. DNA methylation and gene activity. Annu Rev Biochem 1983; 52:93-124.

2 Clark SJ, Harrison J, Frommer M. CpNpG methylation in mammalian cells. Nat Genet 1995; 10:20-7.

3 Ramsahoye BH, Biniszkiewicz D, Lyko F, et al. Non-CpG methylation is prevalent in embryonic stem cells and may be mediated by DNA methyltransferase 3a. Proc Natl Acad Sci U S A 2000; 97:5237-42.

4 Lorincz MC, Dickerson DR, Schmitt M, Groudine M. Intragenic DNA methylation alters chromatin structure and elongation efficiency in mammalian cells. Nat Struct Mol Biol 2004; 11:106875.

5 Bestor TH. Cloning of a mammalian DNA methyltransferase. Gene 1988; 74:9-12.

6 Okano M, Bell DW, Haber DA, Li E. DNA methyltransferases DNMT3a and DNMT3b are essential for de novo methylation and mammalian development. Cell 1999; 9:9247-57.

7 Pradhan S, Bacolla A, Wells RD, Roberts RJ. Recombinant human DNA (cytosine-5) methyltransferase. I. Expression, purification, and comparison of de novo and maintenance methylation. J Biol Chem 1999; 274:33002-10.

8 Yoder JA, Soman NS, Verdine GL, Bestor TH. DNA (cytosine5)-methyltransferases in mouse cells and tissues. Studies with a mechanism-based probe. J Mol Biol 1997; 270:385-95.

9 Leonhardt H, Page AW, Weier HU, Bestor TH. A targeting sequence directs DNA methyltransferase to sites of DNA replication in mammalian nuclei. Cell 1992; 71:865-73.

10 Rountree MR, Bachman KE, Baylin SB. DNMT1 binds HDAC2 and a new co-repressor, DMAP1, to form a complex at replication foci. Nat Genet 2000; 25:338-42.

11 Fuks F, Burgers WA, Brehm A, et al. DNA methyltransferase Dnmt1 associates with histone deacetylase activity. Nat Genet 2000; 24:88-91.

12 Robertson KD, Ait-Si-Ali S, Yokochi T, et al. DNMT1 forms a complex with Rb, E2F1 and HDAC1 and represses transcription from E2F-responsive promoters. Nat Genet 2000; 25:33842.

www.cell-research.com | Cell Research, 15(9):679-690, Sep 2005
13 Li E, Bestor TH, Jaenisch R. Targeted mutation of the DNA methyltransferase gene results in embryonic lethality. Cell 1992; 69:915-26.

14 Lei H, Oh SP, Okano M, et al. De novo DNA cytosine methyltransferase activities in mouse embryonic stem cells. Development 1996; 122:3195-205.

$15 \mathrm{Li}$ E, Beard C, Jaenisch R. Role for DNA methylation in genomic imprinting. Nature 1993; 366:362-5.

16 Chen RZ, Pettersson U, Beard C, Jackson-Grusby L, Jaenisch R. DNA hypomethylation leads to elevated mutation rates. Nature 1998; 395:89-93.

17 Laird PW, Jackson-Grusby L, Fazeli A, et al. Suppression of intestinal neoplasia by DNA hypomethylation. Cell 1995; 81: 197-205.

18 Walsh CP, Bestor TH. Cytosine methylation and mammalian development. Genes Dev 1999; 13:26-34.

19 Kato M, Miura A, Bender J, et al. Role of CG and non-CG methylation in immobilization of transposons in Arabidopsis. Curr Biol 2003; 13:421-6.

20 Bourc'his D, Bestor TH. Meiotic catastrophe and retrotransposon reactivation in male germ cells lacking Dnmt3L. Nature 2004; 431:96-99.

21 Gaudet F, Hodgson JG, Eden A, et al. Induction of tumors in mice by genomic hypomethylation. Science 2003; 300:489-92.

22 Okano M, Xie S, Li E. Cloning and characterization of a family of novel mammalian DNA (cytosine-5) methyltransferases. Nat Genet 1998; 19:219-20.

$23 \mathrm{Xu}$ GL, Bestor TH, Bourc'his D, et al. Chromosome instability and immunodeficiency syndrome caused by mutations in a DNA methyltransferase gene. Nature 1999; 402:187-91.

24 Chen T, Ueda Y, Dodge JE, Wang Z, Li E. Establishment and maintenance of genomic methylation patterns in mouse embryonic stem cells by Dnmt3a and Dnmt3b. Mol Cell Biol 2003; 23: 5594-605.

25 Lyko F, Ramsahoye BH, Kashevsky H, et al. Mammalian (cytosine-5) methyltransferases cause genomic DNA methylation and lethality in Drosophila. Nat Genet 1999; 23:363-66.

26 Aapola U, Lyle R, Krohn K, Antonarakis SE, Peterson P. Isolation and initial characterization of the mouse Dnmt31 gene. Cytogenet Cell Genet 2001; 92:122-6.

27 Hata K, Okano M, Lei H, Li E. Dnmt3L cooperates with the Dnmt3 family of de novo DNA methyltransferases to establish maternal imprints in mice. Development 2002; 129:1983-93.

28 Bourc'his D, Xu GL, Lin CS, Bollman B, Bestor TH. Dnmt3L and the establishment of maternal genomic imprints. Science 2001; 294:2536-39.

29 Chedin F, Lieber MR, Hsieh CL. The DNA methyltransferaselike protein DNMT3L stimulates de novo methylation by Dnmt3a. Proc Natl Acad Sci U S A 2002; 99:16916-21.

30 Rhee I, Bachman KE, Park BH, et al. DNMT1 and DNMT3b cooperate to silence genes in human cancer cells. Nature 2002; 416:552-6.

31 Liang G, Chan MF, Tomigahara Y, et al. Cooperativity between DNA methyltransferases in the maintenance methylation of repetitive elements. Mol Cell Biol 2002; 22:480-91.

32 Kim GD, Ni J, Kelesoglu N, Roberts RJ, Pradhan S. Co-operation and communication between the human maintenance and de novo DNA (cytosine-5) methyltransferases. EMBO J 2002; 21 : 4183-95. 
33 Bird AP. CpG-rich islands and the function of DNA methylation. Nature 1986; 321:209-213.

34 Takai D, Jones PA. Comprehensive analysis of $\mathrm{CpG}$ islands in human chromosomes 21 and 22. Proc Natl Acad Sci U S A2002; 99:3740-5.

35 Macleod D, Charlton J, Mullins J, Bird AP. Sp1 sites in the mouse aprt gene promoter are required to prevent methylation of the CpG island. Genes Dev 1994; 8:2282-92.

36 Mummaneni P, Walker KA, Bishop PL, Turker MS. Epigenetic gene inactivation induced by a cis-acting methylation center. $\mathrm{J}$ Biol Chem 1995; 270:788-92.

37 Zardo G, Caiafa P. The unmethylated state of $\mathrm{CpG}$ islands in mouse fibroblasts depends on the poly(ADP-ribosyl)ation process. J Biol Chem 1998; 273:16517-20.

38 Pradhan S, Kim GD. The retinoblastoma gene product interacts with maintenance human DNA (cytosine-5) methyltransferase and modulates its activity. EMBO J 2002; 21:779-88.

39 Di Croce L, Raker VA, Corsaro M, et al. Methyltransferase recruitment and DNA hypermethylation of target promoters by an oncogenic transcription factor. Science 2002; 295:1079-82.

40 Cervoni N, Detich N, Seo SB, Chakravarti D, Szyf M. The oncoprotein Set/TAF-1beta, an inhibitor of histone acetyltransferase, inhibits active demethylation of DNA, integrating DNA methylation and transcriptional silencing. J Biol Chem 2002; 277:25026-31.

41 Larsen F, Gundersen G, Lopez R, Prydz H. CpG islands as gene markers in the human genome. Genomics 1992; 13:1095-107.

42 Smit AF, Riggs AD. Tiggers and DNA transposon fossils in the human genome. Proc Natl Acad Sci U S A 1996; 93:1443-8.

43 Hirochika H, Okamoto $H$, Kakutani T. Silencing of retrotransposons in arabidopsis and reactivation by the ddm 1 mutation. Plant Cell 2000; 12:357-69.

44 Takahara T, Ohsumi T, Kuromitsu J, et al. Dysfunction of the Orleans reeler gene arising from exon skipping due to transposition of a full-length copy of an active L1 sequence into the skipped exon. Hum Mol Genet 1996; 5:989-93.

45 Razin A, Cedar H. DNA methylation and embryogenesis. EXS 1993; 64:343-57.

46 Kafri T, Ariel M, Brandeis M, et al. Developmental pattern of gene-specific DNA methylation in the mouse embryo and germ line. Genes Dev 1992; 6:705-14.

47 Young LE, Beaujean N. DNA methylation in the preimplantation embryo: the differing stories of the mouse and sheep. Anim Reprod Sci 2004; 82-83:61-78.

48 Tremblay KD, Saam JR, Ingram RS, Tilghman SM, Bartolomei MS. A paternal-specific methylation imprint marks the alleles of the mouse H19 gene. Nat Genet 1995; 9:407-13.

49 Kass SU, Landsberger N, Wolffe AP. DNA methylation directs a time-dependent repression of transcription initiation. Curr Biol 1997; 7:157-65.

50 Song F, Smith JF, Kimura MT, et al. Association of tissuespecific differentially methylated regions (TDMs) with differential gene expression. Proc Natl Acad Sci U S A 2005; 102: 3336-41.

51 Ching TT, Maunakea AK, Jun P, et al. Epigenome analyses using BAC microarrays identify evolutionary conservation of tissue-specific methylation of SHANK3. Nat Genet 2005; 37: 645-651.

52 Iguchi-Ariga SM, Schaffner W. CpG methylation of the cAMP- responsive enhancer/promoter sequence TGACGTCA abolishes specific factor binding as well as transcriptional activation. Genes Dev 1989; 3:612-9.

53 Bird AP, Wolffe AP. Methylation-induced repression-belts, braces, and chromatin. Cell 1999; 99:451-4.

54 Guy J, Hendrich B, Holmes M, Martin JE, Bird A. A mouse Mecp2-null mutation causes neurological symptoms that mimic Rett syndrome. Nat Genet 2001; 27:322-6.

55 Jorgensen HF, Ben Porath I, Bird AP. Mbd1 is recruited to both methylated and nonmethylated CpGs via distinct DNA binding domains. Mol Cell Biol 2004; 24:3387-95.

$56 \mathrm{Ng} \mathrm{HH}$, Zhang Y, Hendrich B, et al. MBD2 is a transcriptional repressor belonging to the $\mathrm{MeCP} 1$ histone deacetylase complex. Nat Genet 1999; 23:58-61.

57 Hendrich B, Guy J, Ramsahoye B, Wilson VA, Bird A. Closely related proteins MBD2 and MBD3 play distinctive but interacting roles in mouse development. Genes Dev 2001; 15:710-23.

58 Yoon JH, Iwai S, O'Connor TR, Pfeifer GP. Human thymine DNA glycosylase (TDG) and methyl-CpG-binding protein 4 (MBD4) excise thymine glycol (Tg) from a Tg:G mispair. Nucleic Acids Res 2003; 31:5399-404.

59 Prokhortchouk A, Hendrich B, Jorgensen H, et al. The p120 catenin partner Kaiso is a DNA methylation-dependent transcriptional repressor. Genes Dev 2001; 15:1613-8.

60 Ruzov A, Dunican DS, Prokhortchouk A, et al. Kaiso is a genome-wide repressor of transcription that is essential for amphibian development. Development 2004; 131:6185-94.

61 Yoon HG, Chan DW, Reynolds AB, Qin J, Wong J. N-CoR mediates DNA methylation-dependent repression through a methyl CpG binding protein Kaiso. Mol Cell 2003; 12:723-4.

62 Jones PL, Veenstra GI, Wade PA, et al. Methylated DNA and $\mathrm{MeCP} 2$ recruit histone deacetylase to repress transcription. Nat Genet 1998; 19:187-91.

63Nan X, Ng HH, Johnson CA, Turner BM, Bird A. Transcriptional repression by the methyl-CpG-binding protein $\mathrm{MeCP} 2$ involves a histone deacetylase complex. Nature 1998; 393:386-9.

64 Wade PA, Gegonne A, Jones PL, et al. Mi-2 complex couples DNA methylation to chromatin remodelling and histone deacetylation. Nat Genet 1999; 23:62-6.

65 Santoro R, Li J, Grummt I. The nucleolar remodeling complex NoRC mediates heterochromatin formation and silencing of ribosomal gene transcription. Nat Genet 2002; 32:393-6.

66 Zhang Y, Ng HH, Erdjument-Bromage H, et al. Analysis of the NuRD subunits reveals a histone deacetylase core complex and a connection with DNA methylation. Genes Dev 1999; 13:192435 .

67 Feng Q, Zhang Y. The MeCP1 complex represses transcription through preferential binding, remodeling, and deacetylating methylated nucleosomes. Genes Dev 2001; 15:827-32.

68 Zhang Y, Reinberg D. Transcription regulation by histone methylation: interplay between different covalent modifications of the core histone tails. Genes Dev 2001; 15:2343-60.

69 Kouzarides T. Histone acetylases and deacethylases in cell proliferation. Curr Opin Genet Dev 1999; 9:40-8.

70 Kouzarides T. Histone methylation in transcriptional control. Curr Opin Genet Dev 2002; 12:198-209.

71 Hake SB, Xiao A, Allis CD. Linking the epigenetic 'language' of covalent histone modifications to cancer. Br J Cancer 2004; 90: 761-9. 
72 Tamaru H, Selker EU. A histone H3 methyltransferase controls DNA methylation in Neurospora crassa. Nature 2001; 414:27783.

73 Jackson JP, Lindroth AM, Cao X, Jacobsen SE. Control of CpNpG DNA methylation by the KRYPTONITE histone H3 methyltransferase. Nature 2002; 416:556-60.

74 Lehnertz B, Ueda Y, Derijck AA, et al. Suv39h-mediated histone H3 lysine 9 methylation directs DNA methylation to major satellite repeats at pericentric heterochromatin. Curr Biol 2003; 13: 1192-200.

75 Wassenegger M. RNA-directed DNA methylation. Plant Mol Biol 2000; 43:203-20.

76 Aufsatz W, Mette MF, van der WJ, Matzke AJ, Matzke M. RNA-directed DNA methylation in Arabidopsis. Proc Natl Acad Sci U S A 2002; 99 Suppl 4:16499-506.

77 Jones L, Ratcliff F, Baulcombe DC. RNA-directed transcriptional gene silencing in plants can be inherited independently of the RNA trigger and requires Met1 for maintenance. Curr Biol 2001; 11:747-57.

78 Melquist S, Bender J. Transcription from an upstream promoter controls methylation signaling from an inverted repeat of endogenous genes in Arabidopsis. Genes Dev 2003; 17:2036-47.

79 Morris KV, Chan SW, Jacobsen SE, Looney DJ. Small interfering RNA-induced transcriptional gene silencing in human cells. Science 2004; 305: 1289-92.

80 Kawasaki H, Taira K. Induction of DNA methylation and gene silencing by short interfering RNAs in human cells. Nature 2004; 431:211-7.

81 Bao N, Lye KW, Barton MK. MicroRNA binding sites in Arabidopsis class III HD-ZIP mRNAs are required for methylation of the template chromosome. Dev Cell 2004; 7:653-62.

82 Ronemus M, Martienssen R. RNA interference: methylation mystery. Nature 2005; 433:472-3.

83 Costello JF, Plass C. Methylation matters. J Med Genet 2001; 38:285-303.

84 Belinsky SA. Gene-promoter hypermethylation as a biomarker in lung cancer. Nat Rev Cancer 2004; 4:707-17.

85 Holst CR, Nuovo GJ, Esteller M, et al. Methylation of p16 (INK4a) promoters occurs in vivo in histologically normal human mammary epithelia. Cancer Res 2003; 63:1596-601.

86 Gilbert J, Gore SD, Herman JG, Carducci MA. The clinical application of targeting cancer through histone acetylation and hypomethylation. Clin Cancer Res 2004; 10:4589-96.

87 Ehrlich M. DNA methylation in cancer: too much, but also too little. Oncogene 2002; 21:5400-13.

88 Feinberg AP, Vogelstein B. Hypomethylation distinguishes genes of some human cancers from their normal counterparts. Nature 1983; 301:89-92.

89 Eden A, Gaudet F, Waghmare A, Jaenisch R. Chromosomal instability and tumors promoted by DNA hypomethylation. Science $2003 ; \mathbf{3 0 0}: 455$.

90 Feinberg AP. Imprinting of a genomic domain of $11 \mathrm{p} 15$ and loss of imprinting in cancer: an introduction. Cancer Res 1999; 59: 1743-46s.

91 Lippman Z, Gendrel AV, Black M, et al. Role of transposable elements in heterochromatin and epigenetic control. Nature 2004; 430:471-6.

92 Bernstein E, Allis CD. RNA meets chromatin. Genes Dev 2005; 19:1635-55.
93 Ehrlich M. The controversial denouement of vertebrate DNA methylation research. Biochemistry (Mosc ) 2005; 70:568-75.

94 Gonzalgo ML, Jones PA. Mutagenic and epigenetic effects of DNA methylation. Mutat Res 1997; 386:107-18.

95 Schmutte C, Yang AS, Beart RW, Jones PA. Base excision repair of $\mathrm{U}: \mathrm{G}$ mismatches at a mutational hotspot in the p53 gene is more efficient than base excision repair of $\mathrm{T}: \mathrm{G}$ mismatches in extracts of human colon tumors. Cancer Res 1995; 55:3742-6.

96 Zardo G, Tiirikainen MI, Hong C, et al. Integrated genomic and epigenomic analyses pinpoint biallelic gene inactivation in tumors. Nat Genet 2002; 32:453-8.

97 Costello JF, Fruhwald MC, Smiraglia DJ, et al. Aberrant CpGisland methylation has non-random and tumour-type-specific patterns. Nat Genet 2000; 24:132-8.

98 Egger G, Liang G, Aparicio A, Jones PA. Epigenetics in human disease and prospects for epigenetic therapy. Nature 2004; 429: 457-63.

99 Feinberg AP, Tycko B. The history of cancer epigenetics. Nat Rev Cancer 2004; 4:143-53.

100 Chambon P. A decade of molecular biology of retinoic acid receptors. FASEB J 1996; 10:940-54.

101 Kastner P, Chan S. Function of RARalpha during the maturation of neutrophils. Oncogene 2001; 20:7178-85.

102Mangelsdorf DJ, Evans RM. The RXR heterodimers and orphan receptors. Cell 1995; 83:841-50.

103 Dilworth FJ, Chambon P. Nuclear receptors coordinate the activities of chromatin remodeling complexes and coactivators to facilitate initiation of transcription. Oncogene 2001; 20:304754.

104Glass CK, Rosenfeld MG. The coregulator exchange in transcriptional functions of nuclear receptors. Genes Dev 2000; 14: 121-41.

105 Melnick A, Licht JD. Deconstructing a disease: RAR $\alpha$, its fusion partners, and their roles in the pathogenesis of acute promyelocytic leukemia. Blood 1999; 93:3167-215.

106 Nervi C, Poindexter EC, Grignani F, et al. Characterization of the PML-RARa chimeric product of the acute promyelocytic leukemia specific $\mathrm{t}(15 ; 17)$ translocation. Cancer Res 1992; 52:36873692.

107Minucci S, Maccarana M, Cioce M, et al. Oligomerization of RAR and AML1 transcription factors as a novel mechanism of oncogenic activation. Mol Cell 2000; 5:811-20.

108 Grignani F, De Matteis S, Nervi C, et al. Fusion proteins of the retinoic acid receptor-a recruit histone deacetylase in promyelocytic leukaemia. Nature 1998; 391:815-18.

109Lin RJ, Nagy L, Inoue S, et al. Role of the histone deacetylase complex in acute promyelocytic leukaemia. Nature 1998; 391: 811-4.

$110 \mathrm{He}$ LZ, Guidez F, Tribioli C, et al. Distinct interactions of PMLRARalpha and PLZF-RARalpha with co-repressors determine differential responses to RA in APL. Nat Genet 1998; 18:12635.

111 Warrell RPJ, He LZ, Richon V, Calleja E, Pandolfi PP. Therapeutic targeting of transcription in acute promyelocytic leukemia by use of an inhibitor of histone deacetylase. J Natl Cancer Inst 1998; 90:1621-5.

112Fazi F, Travaglini L, Carotti D, et al. Retinoic acid targets DNAmethyltransferases and histone deacetylases during APL blast differentiation in vitro and in vivo. Oncogene 2005; 24:1820- 
30.

113 Sanz MA, Tallman MS, Lo-Coco F. Tricks of the trade for the appropriate management of newly diagnosed acute promyelocytic leukemia. Blood 2005; 105:3019-25.

114Mizuno S, Chijiwa T, Okamura T, et al. Expression of DNA methyltransferases DNMT1, 3A, and 3B in normal hematopoiesis and in acute and chronic myelogenous leukemia. Blood 2001; 97:1172-9.

115Fenaux P, Degos L. Differentiation therapy for acute promyelocytic leukemia. N Engl J Med 1997; 337:1076-7.

116 Gelmetti V, Zhang J, Fanelli M, et al. Aberrant recruitment of the nuclear receptor corepressor-histone deacetylase complex by the acute myeloid leukemia fusion partner ETO. Mol Cell Biol 1998; 18:7185-92.

117Redner RL, Wang J, Liu JM. Chromatin remodelling and leukemia: new therapeutic paradigms. Blood 1999; 94:417-28.

118Carapeti M, Aguiar RC, Goldman JM, Cross NC. A novel fusion between MOZ and the nuclear receptor coactivator TIF2 in acute myeloid leukemia. Blood 1999; 91:3127-33.

119Borrow J, Stanton VP, Andresen JM, et al. The translocation $\mathrm{t}$
$(8 ; 16)(\mathrm{p} 11 ; \mathrm{p} 13)$ of acute myeloid leukemia fuses a putative acetyltransferase to the CREB-binding protein. Nat Genet 1996; 14:33-41.

120 Sobulo O, Borrow J, Tomek R, et al. MLL is fused to CBP, a histone acetyltransferase, in therapy-related acute myeloid leukemia with a $t(11 ; 16)(q 23 ; p 13.3)$. Proc Natl Acad Sci U S A 1997; 94:8732-7.

121 Ida K, Kitabayashi I, Taky T, et al. Adenovirusn E1A-associated protein $\mathrm{p} 300$ is involved in acute myeloid leukaemia with $\mathrm{t}(11$; 22). Blood 1997; 90:4699-704

122 Lavau C, Du C, Thirman M, Zeleznik-Le N. Chromatin-related properties of CBP fused to MLL generate a myelodysplasticlike syndrome that evolves into myeloid leukemia. EMBO J 2000; 19:4655-64.

123 Ferrara FF, Fazi F, Bianchini A, et al. Histone deacetylase targeted treatment restores retinoic acid signaling and differentiation in acute myeloid leukemia. Cancer Res 2001; 61:2-7.

124 Gottlicher M, Minucci S, Zhu P, et al. Valproic acid defines a novel class of HDAC inhibitors inducing differentiation of transformed cells. EMBO J 2001; 20:6969-78.

Edited by Lu LIANG 